\title{
Indium tin oxide with zwitterionic interfacial design for biosensing applications in complex matrices
}

\author{
Nadia T. Darwish, Yatimah Alias, Sook Mei Khor* \\ Department of Chemistry, Faculty of Science University of Malaya, 50603 Kuala Lumpur, Malaysia
}

\section{A R T I C L E I N F O}

\section{Article history:}

Received 5 September 2014

Received in revised form 15 October 2014

Accepted 28 October 2014

Available online 5 November 2014

\section{Keywords:}

Biosensor

Indium tin oxide

Anti-biofouling coating

Zwitterionic molecules

\begin{abstract}
A B S T R A C T
Biosensing interfaces consisting of linker molecules $\left(\mathrm{COOH}\right.$ or $\left.\mathrm{NH}_{2}\right)$ and charged, antifouling moieties $\left(\left(-\mathrm{SO}^{3-}\right.\right.$ and $\left.\mathrm{N}^{+}(\mathrm{Me})_{3}\right)$ for biosensing applications were prepared for the first time by the in situ deposition of mixtures of aryl diazonium cations on indium tin oxide (ITO) electrodes. A linker molecule is required for the attachment of biorecognition molecules (e.g., antibodies, enzymes, DNA chains, and aptamers) close to the transducer surface. The attached molecules improve the biosensing sensitivity and also provide a short response time for analyte detection. Thus, the incorporation of a linker and antifouling molecules is an important interfacial design for both affinity and enzymatic biosensors. The reductive adsorption behavior and electrochemical measurement were studied for (1) an individual compound and (2) a mixture of antifouling zwitterionic molecules together with linker molecules [combination 1: 4sulfophenyl (SP), 4-trimethylammoniophenyl (TMAP), and 1,4-phenylenediamine (PPD); combination 2: 4-sulfophenyl (SP), 4-trimethylammoniophenyl (TMAP), and 4-aminobenzoic acid (PABA)] of aryl diazonium cations grafted onto an ITO electrode. The mixture ratios of SP:TMAP:PPD and SP:TMAP:PABA that provided the greatest resistance to non-specific protein adsorptions of bovine serum albumin labeled with fluorescein isothiocyanate (BSA-FITC) and cytochrome $c$ labeled with rhodamine B isothiocyanate (RBITC-Cyt $c$ ) were determined by confocal laser scanning microscopy (CLSM). For the surface antifouling study, we used 2-[2-(2-methoxyethoxy) ethoxy]acetic acid (OEG) as a standard control because of its prominent antifouling properties. Surface compositions of combinations 1 and 2 were characterized using X-ray photoelectron spectroscopy (XPS). Field-emission scanning electron microscopy (FE-SEM) was used to characterize the morphology of the grafted films to confirm the even distribution between linker and antifouling molecules grafted onto the ITO surfaces. Combination 1 (SP:TMAP:PPD) with a ratio of $0.5: 1.5: 0.37$ exhibited the best antifouling capability with respect to resisting the nonspecific adsorption of proteins.
\end{abstract}

\section{Introduction}

Biofouling is the accretion of proteins, cells and other biological materials onto a bare electrode surface. For fast clinical screening, immunosensors are designed to detect disease analytes such as nucleic acids, proteins or other disease biomarkers at very low concentrations from complicated sample media [1-5]. Such an accumulation of biofouling substances leads to reduced analyte diffusion and perfusion to the sensor interface, which eventually causes a decrease in sensor response and sensitivity. Polymers such as polyurethane, polyvinyl chloride, poly(dimethylsiloxane) and poly(methacrylate) have been used as antifouling agents for biosensing surfaces, where they provide selective transport

\footnotetext{
* Corresponding author. Tel.: +60 3 79677022x2520; fax: +60 39674193.

E-mail address: naomikhor@um.edu.my (S.M. Khor).
}

of a targeted analyte to the biosensing interface while preventing biofouling components from reaching the electrode surface [6-10]. Although these polymers are exceptionally suitable in clinical monitoring, their functional stability is greatly affected by the biocompatibility of the biosensor materials in contact with the biological sample [11,12]. Poly(ethylene glycol) (PEG) and self-assembled monolayers with oligo(ethylene glycol) chains (OEG-SAMs) have been used to reduce the non-specific adsorption of non-desired proteins [13].

The activity of anti-biofouling coatings in complex samples, e.g., human sera, depends on several factors, including the concentrations and chain lengths of PEG and OEG and the temperature. At $37^{\circ} \mathrm{C}$, the grafted surface can adsorb additional non-specific proteins. Moreover, surfaces grafted with PEG and OEG are prone to auto-oxidation in most biochemically relevant solutions for in vivo studies $[6,14,15]$. Zwitterionic polymers such as phosphorylcholine, sulfobetaine, and carboxy betaine, which contain both 
anionic and cationic groups, have been used as non-fouling materials. Unfortunately, grafting of the long chains of zwitterionic polymers can result in the formation of high-impedance layers and subsequently cause losses to electrode sensitivities. However, short-chain molecules exhibit poor stability [15-17]. Gooding and co-workers investigated zwitterionic phenyl derivative layers bearing charged moieties, such as $-\mathrm{SO}_{3}{ }^{-}$and $-\mathrm{N}^{+}(\mathrm{Me})_{3}$, deposited onto glassy carbon surfaces as an alternative to OEG phenyl layers and observed that the grafted zwitterionic phenyl derivative layers offer better chemical stability and a shorter electron transfer pathway to the transducer surface [15]. Zwitterionic phenyl layers, such as OEG alkanethiol SAMs, have been proposed as effective alternatives to conventional antifouling molecules because of their antifouling properties. Such coatings do not completely passivate the electrode surface and therefore allow Faradaic electrochemistry. Hence, they can be utilized in biosensing applications.

Indium tin oxide (ITO) is a biosensing substrate of increasing importance in the field of biomaterials because of its combined properties of transparency, conductivity and biocompatibility [18]. Very few studies have examined the use of antifouling molecules on ITO for bioassay contexts. To date, only hydrophilic polyacrylic acid brushes [19] and zwitterionic polymer brushes [20] have been investigated as antifouling agents. However, in these studies, only BSA-FITC was used to evaluate the antifouling capability. The use of only a single protein may not be representative of complex media, such as blood plasma and serum that contain a variety of proteins. Moreover, a different study grafted zwitterionic polymers onto the ITO using surface-initiated atom transfer radical polymerization (SI-ATRP). Although the obtained organic coatings were considered stable in aqueous solution, the technique was time consuming and had moderate throughput [21]. The literature contains no accounts of research related to the capability of these antifouling moieties after they have been modified with linker molecules, such as 1,4-phenylenediamine or 4-aminobenzoic acid. A linker molecule is required for the attachment of biorecognition molecules (e.g., antibodies, enzymes, DNA, or aptamers) close to the transducer surface.

The incorporation of zwitterionic phenyl layers with a linker molecule is useful for improving the selectivity of the biosensing interface, which is resistant to the nonspecific adsorption of proteins, and for providing a shorter response time for specific analyte detection (e.g., low impedance due to a shorter electron pathway). Therefore, one objective of this study was to analyze the electrochemical deposition behavior of OEG when deposited onto ITO electrodes compared with the behaviors of a single molecule and two mixed layer coatings comprising 4-sulfophenyl (SP), 4trimethylammoniophenyl (TMAP), 1,4-phenylenediamine (PPD), and 4-aminobenzoic acid (BAPA): mixed layers 1 (SP:TMAP:PPD) and mixed layers 2 (SP:TMAP:BAPA). The second objective of this study was to investigate the capability of an ITO surface modified with the aforementioned molecules to repel both a negatively charged protein (BSA-FITC) and a positively charged protein (RBITC-Cyt $c$ ). These example proteins were used to represent proteins contained in real samples. ITO was modified using different ratios of antifouling molecules (SP or TMAP) to linker molecules (PPD or BAPA). We examined the antifouling properties toward the nonspecific absorption of fluorescently labeled proteins using confocal laser scanning microscopy (CLSM). The third objective of this study was to characterize the modified surface with the lowest protein adsorption level to both BSA-FITC and RBITC-Cyt $c$ by using $\mathrm{X}$-ray photoelectron spectroscopy (XPS) to confirm the existence of SP, TMAP, PPD or PABA moieties on the grafted ITO. The final objective of this study was to examine the surface morphology of the grafted films, where the surface distribution of SP:TMAP:PPD could be indicated by $\mathrm{NH}$-gold nanoparticles and characterized using field-emission scanning electron microscopy (FE-SEM).

\section{Experimental methods}

\subsection{Reagents and materials}

ITO coated glass slide (surface resistivity $\leq 7 \Omega / \mathrm{sq}, L \times W \times$ thickness $406 \mathrm{~mm} \times 355 \mathrm{~mm} \times 0.7 \mathrm{~mm}$, sheet) was purchased from (Sanyo, Japan). 4-sulfophenyl, 1,4-phenylenediamine, 4aminobenzoic acid, sodium nitrite $\left(\mathrm{NaNO}_{2}\right)$, potassium chloride $(\mathrm{KCl})$, potassium dihydrogen phosphate $\left(\mathrm{KH}_{2} \mathrm{PO}_{4}\right)$, potassium phosphate dibasic $\left(\mathrm{K}_{2} \mathrm{HPO}_{4}\right)$, hydrochloric acid $(\mathrm{HCl})$, potassium ferricyanide $\left(\mathrm{K}_{3} \mathrm{Fe}(\mathrm{CN})_{6}\right)$, hexamine ruthenium(III) chloride $\left(\mathrm{Ru}\left(\mathrm{NH}_{3}\right)_{6} \mathrm{Cl}_{3}\right)$, sodium carbonate $\left(\mathrm{Na}_{2} \mathrm{CO}_{3}\right)$ with $99 \%$ purity, $20 \mathrm{~nm}$ gold nanoparticles, sodium bicarbonate $\left(\mathrm{NaHCO}_{3}\right)$, rhodamine $B$ isothiocyanate, bovine serum albumin-fluorescein isothiocyanate, cytochrome $c$ (from bovine heart) and 2-[2-(2methoxyethoxy)ethoxy]acetic acid (OEG) were purchased from Sigma-Aldrich (USA). 4-Trimethylammoniophenyl (TMAP) was obtained from Acros (USA). All solutions were prepared using Milli$Q$ water $(18 \mathrm{M} \Omega / \mathrm{cm})$ (Purelab, US).

\subsection{ITO electrode surface modification}

ITO substrates were soaked and cleaned in an ultrasonicator using dichloromethane and then $99 \%$ methanol for 10 min each, followed by treatment with $0.5 \mathrm{M} \mathrm{K}_{2} \mathrm{CO}_{3}$ in a 3:1 methanol:Milli- $Q$ water mixture for $30 \mathrm{~min}$ under sonication to remove any residual organic contaminants. The ITO substrates were then rinsed with copious amounts of Milli- $Q$ water, dried and stored in an nitrogenfilled container [22]. In the first experiment, the ITO electrodes were modified with only a single compound: SP, PPD, BAPA, or TMAP. In the second experiment, the ITO electrodes were modified with a combination of SP:TMAP:PPD at different molar ratios: $1: 1: 0.37(\operatorname{mix} 1), 1.5: 0.5: 0.37(\operatorname{mix} 2)$ and $0.5: 1.5: 0.37(\operatorname{mix} 3)$. In the third experiment, the ITO electrodes were modified with a combination of SP:TMAP:PABA at different molar ratios: 0.8:1:0.2 (mix 1), $1: 1: 0.1(\operatorname{mix} 2)$ and $0.5: 1: 0.2(\operatorname{mix} 3)$. For the control experiment, OEG was used as the antifouling molecule on the ITO slides. The OEG was prepared by the electrodeposition of a mixed layer of 4aminobenzoic acid ( $8 \mathrm{mM}$ ) and 1,4-phenyldiamine ( $2 \mathrm{mM})$ on ITO slides. The electrode interface was fabricated using carbodiimide chemistry: we linked the OEG to the $\mathrm{COOH}$ moieties of PPD amine groups by incubating the modified surface in an ethanol solution containing $10 \mathrm{mM}$ OEG and $40 \mathrm{mM}$ DCC for $6 \mathrm{~h}$ at room temperature. In all of the aforementioned experiments, in situ methods were used in which aryl diazonium cations were electrochemically reduced with two times the molar amount of $\mathrm{NaNO}_{2}$ in $0.05 \mathrm{M} \mathrm{HCl}$ The solution was purged with nitrogen gas for 20 min before the surface modification. The electrodeposition was performed using cyclic voltammetry at $-0.6 \mathrm{~V}$ to $0.2 \mathrm{~V}$; the electrodes were scanned for 5 cycles at $100 \mathrm{mV} \mathrm{s}^{-1}[23-25]$.

\subsection{Surface passivation study via electrochemical measurements}

All voltammetry measurements were performed using an AutoLabIII potentiostat (Metrohm AutoLab, Netherlands) and a conventional three-electrode system comprising an ITO working electrode, a platinum wire as the auxiliary electrode, and $\mathrm{Ag} / \mathrm{AgCl}$ $(3.0 \mathrm{M} \mathrm{NaCl})$ as the reference electrode. All potentials were reported relative to the $\mathrm{Ag} / \mathrm{AgCl}$ reference electrode at room temperature. The surface blocking studies on the modified sensing interface were performed in two aqueous solutions containing different redox probes. $\left[\mathrm{Fe}(\mathrm{CN})_{6}\right]^{4-/ 3-},\left[\mathrm{Ru}\left(\mathrm{NH}_{3}\right)_{6}\right]^{3+}$ and $\mathrm{CV}$ were used to probe the integrity of the functionalized layers on the electrode surfaces. The respective redox-active solution $(1 \mathrm{mM})$ was composed of $0.05 \mathrm{M}$ $\mathrm{KCl}, 0.05 \mathrm{M} \mathrm{K}_{2} \mathrm{HPO}_{4}$, and $0.05 \mathrm{M} \mathrm{KH}_{2} \mathrm{PO}_{4}$ and was adjusted to $\mathrm{pH}$ 7.0. ITOs modified with SP:TMAP:PPD (mix 3 ) or SP:TMAP:PABA (mix 
3) were characterized using XPS. The XPS spectra were analyzed using the Casa XPS software (version 2.3.15).

\subsection{Conjugation of cytochrome $c$ to rhodamine B isothiocyanate}

The fluorescent dye rhodamine B was conjugated with Cyt $c$ according to the method reported by Gui et al. [26]. Ten milligrams of Cyt $c$ was dissolved in $1 \mathrm{~mL}$ of $0.1 \mathrm{M}$ sodium carbonatebicarbonate buffer ( $\mathrm{pH} 9.05$ ), and $0.25 \mathrm{~mL}$ of $10 \mathrm{mg} / \mathrm{mL}$ RBITC in DMSO was subsequently added to obtain a fivefold molar excess of Cyt $c$ relative to RBITC. The tube containing the mixture solution was wrapped with aluminum foil and incubated in the dark for $1 \mathrm{~h}$ under continuous magnetic stirring. The mixture was filtered through a $0.22 \mu \mathrm{m}$ pore size syringe filter (non-sterile, $13 \mathrm{~mm}$ diameter; Merck Millipore, Germany), and the clear conjugated solution was subsequently transferred to an Amicon Ultra-15 centrifugal filter device ( $3 \mathrm{kDa} M W$ cut-off, EMD Millipore, USA). The capped filter device was placed in the swinging bucket rotor of a centrifuge and spun for $10 \mathrm{~min}$ at $4000 \mathrm{rpm}$. The separated solution in the upper reservoir of the filter device contained RBITC-Cyt $c$, which was then characterized by UV-vis and fluorescence spectroscopy. The RBITC-Cyt $c$ solution was stored at $-20^{\circ} \mathrm{C}$ until use. The functionalized ITO slides were stored in clean Petri dishes before the antifouling study.

For the antifouling study, the modified ITO electrode was immersed in an adequate amount of either $1 \mathrm{mg} / \mathrm{mL}$ BSA-FITC (an example of a negatively charged protein) or $2 \mathrm{mg} / \mathrm{mL}$ RBITC-Cyt $c$ (an example of a positively charged protein); both immersion solutions were prepared using PBS buffer, $\mathrm{pH}$ 7.4. The Petri dishes were kept in darkness for $1 \mathrm{~h}$, and the slides were subsequently transferred to another Petri dish that contained only PBS. The ITO slides were soaked for $7 \mathrm{~min}$ to remove weakly adsorbed proteins from the surfaces of the slides. Afterward, the slides were washed with Milli- $Q$ water to remove any remaining residues. The fluorescent-labeled-protein-adsorbed surfaces were dried under flowing nitrogen. The samples were mounted with $50 \%$ aqueous glycerol solution and covered with $22 \times 60 \mathrm{~mm}^{2}$ microscope cover slides (Superior Marienfeld, Germany). The dried samples were mounted face-down onto a cover glass $\left(22 \times 60 \mathrm{~mm}^{2}\right.$, No. 1, Paul Marienfeld, GmbH \& Co. KG, Germany) with $50 \%$ aqueous glycerol solution as the mounting agent. For each type of modified surface, three replicates were tested. Five images were captured under $10 \times$ magnification for survey studies. Fifteen images were captured under $63 \times$ magnification, and their mean gray values were calculated to determine the fluorescence of the adsorbed proteins. The mean gray values were compared with different surface types.

\subsection{Surface antifouling study via confocal laser fluorescent microscope imaging}

BSA-FITC and RBITC-Cyt $c$ proteins adsorbed onto functionalized electrode surfaces were measured using a confocal laser fluorescence microscope (Leica Microsystems Pty, Ltd., USA) equipped with an argon laser (at $29 \%$ power) and operated in the xyz scan mode (step size $0.04 \mu \mathrm{m})$. Images $\left(1024 \times 1024 \mathrm{px}^{2}\right)$ were captured at 8-bit resolution using the Las AF software (Origin). The imaging was carried out using two objective lenses: an HCX PL APO CS $10.0 \times 0.40$ Dry UV and an HCX PL APO lambda blue $63.0 \times 1.40$ OIL UV. The gray value represents the fluorescence intensity. Captured TIF-format images were further analyzed using Image (IJ-1.48g. jar) software to determine the mean gray value after background subtraction. For reference, the emission bandwidth of BSA-FITC ranges from $506 \mathrm{~nm}$ to $575 \mathrm{~nm}$. In the case of RBITC-Cyt $c$, the bandwidth ranges from $524 \mathrm{~nm}$ to $594 \mathrm{~nm}$.

\subsection{ITO surface characterization via XPS analysis}

Spectra were obtained using XPS (AXIS Ultra DLD spectrometer with a hemispherical analyzer, a multichannel detector and a

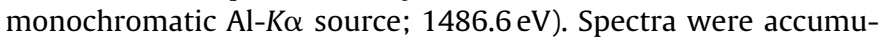
lated at a take-off angle of $90^{\circ}$ with a $0.9 \mathrm{~mm}^{2}$ spot size at a pressure of less than $1 \times 10^{-8}$ mbar. Survey scans $(0-1000 \mathrm{eV})$ were carried out at a $1.0 \mathrm{eV}$ step size, $100 \mathrm{~ms}$ dwell time, and $100 \mathrm{eV}$ analyzer pass energy. High-resolution scans (S2p, C1s, N1s) were performed using a $0.1 \mathrm{eV}$ step size, $100 \mathrm{~ms}$ dwell time, and $20 \mathrm{eV}$ pass energy. Binding energies of elements were corrected with reference to the C1s peak of graphitic carbon $(284.4 \mathrm{eV})$.

\subsection{ITO surface characterization via FE-SEM analysis}

Four different types of surfaces were morphologically characterized using a Hitachi SU8000 field-emission scanning electron microscope (Tokyo, Japan): (1) bare ITO, (2) ITO/gold nanoparticles (GNPs), (3) ITO/SP:TMAP:PPD/GNPs, and (4) ITO/SP:TMAP:PABA. The slides were tested at slow scan speeds less than $2 \mathrm{kV}$ and at $50,000 \times$ magnification. The work destination was $2.1 \mathrm{~mm}$ at an accelerating voltage of $2 \mathrm{kV}$. GNPs were used to indicate the presence of PPD terminal amine groups. GNPs were immobilized by immersing ITO/SP:TMAP:PPD and bare ITO in a GNP solution for $3 \mathrm{~h}$ at room temperature [24]. Energy-dispersive X-ray (EDX) was used to characterize the grafted GNPs.

\section{Results and discussion}

\subsection{Reductive adsorption and electrochemical measurement of individual aryl diazonium cations and their mixtures}

The reductive adsorption peaks of SP $(10 \mathrm{mM})$ and PABA $(10 \mathrm{mM})$ appeared at $-0.473 \mathrm{~V}$ and $-0.10 \mathrm{~V}$, respectively. A cyclic voltammogram of SP diazonium cation solution exhibited an irreversible cathodic peak located at $-0.47 \mathrm{~V}$ (Fig. 1(1a)), similar to the $\mathrm{CV}$ results previously reported for glassy carbon surfaces $[27,28]$. A typical cyclic voltammogram of PABA diazonium cation solution showed a single irreversible cathodic peak at $-0.10 \mathrm{~V}$ (Fig. 1(1d)). The cyclic voltammograms for bare ITO in $1 \mathrm{mM}$ $\left[\mathrm{Fe}(\mathrm{CN})_{6}\right]^{4-13-}$ showed a pair of well-defined redox peaks. Electrode surfaces modified with aryl diazonium cations, SP and PABA showed good passivation toward the penetration of negatively charged redox-active species when the modified surface was tested in $\left[\mathrm{Fe}(\mathrm{CN})_{6}\right]^{4-/ 3-}$. However, these surfaces showed weak passivation properties toward the penetration of positively charged redox active species when the surfaces were tested in $1 \mathrm{mM}$ $\left[\mathrm{Ru}\left(\mathrm{NH}_{3}\right)\right]_{6}{ }^{3+}$. This weak passivation was likely a consequence of the negatively charged layer that formed on the ITO electrode surface, which repelled $\left[\mathrm{Fe}(\mathrm{CN})_{6}\right]^{4-/ 3-}$ but allowed the penetration of $\left[\mathrm{Ru}\left(\mathrm{NH}_{3}\right)\right]_{6}{ }^{3+}$ from the solution to the ITO electrode surface. An opposite effect was observed when the ITO was modified with TMAP (Fig. 1(1b)). This positively charged aryl diazonium cation with a reductive adsorption peak at $-0.26 \mathrm{~V}$ allowed $\left[\mathrm{Fe}(\mathrm{CN})_{6}\right]^{4-/ 3-}$ to penetrate through the layer but prevented $\left[\mathrm{Ru}\left(\mathrm{NH}_{3}\right)_{6}\right]^{3+}$ from diffusing closer to the ITO electrode surface. The modification of the ITO surface with TMAP aryl diazonium cation required a high concentration $(10 \mathrm{mM})$ and up to $10 \mathrm{CV}$ scan cycles to passivate the ITO electrode surface. The difficulty in forming a close-packed layer at a low concentration $(1 \mathrm{mM})$ may be a consequence of the steric hindrance caused by three methyl groups of TMAP, which hinders the possibility of a densely packed structure between the TMAP molecules [29]. The electrodeposition of PPD(5 mM; Fig. 1(1c)) indicated a densely packed surface coverage even after two cycles, and the reductive adsorption peak was observed at $-0.36 \mathrm{~V}$. An efficient 

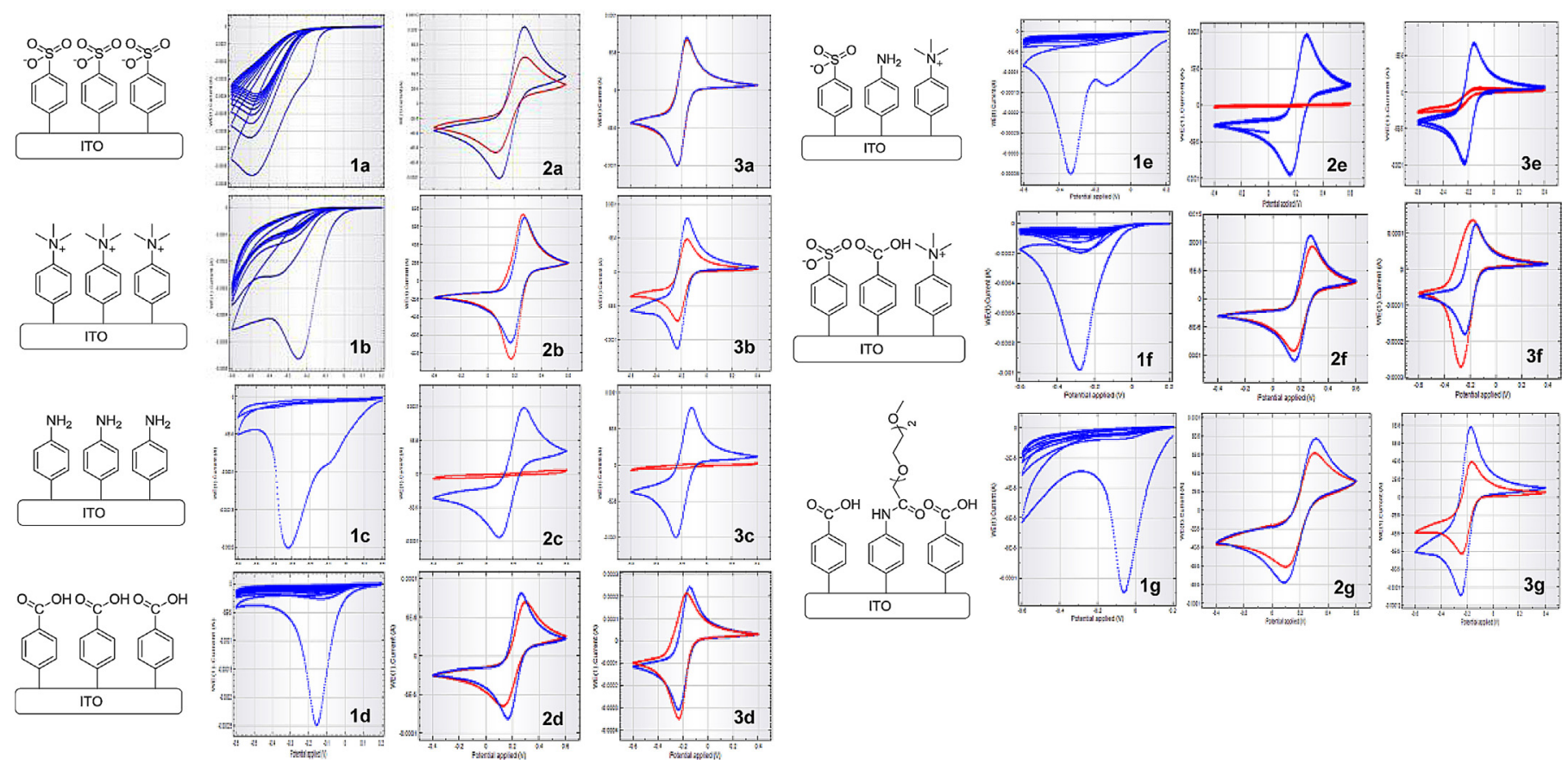

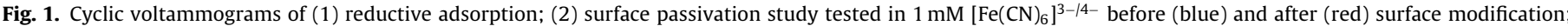

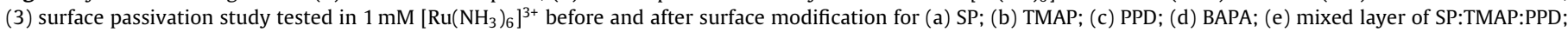

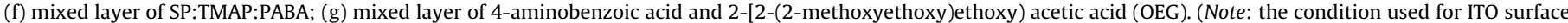

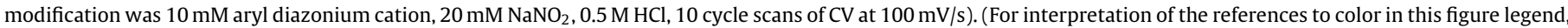
the reader is referred to the web version of this article.)

PPD attachment on the glassy carbon surface has also been reported [30]. This observation may be due to PPD having the fastest diffusion rate and having the lowest molecular weight among the aryl diazonium cations used in this study [31].

The reductive-adsorption cyclic voltammograms of the mixture of SP, TMAP and PPD showed reductive adsorption peaks at approximately $-0.35 \mathrm{~V}$ and $-0.1 \mathrm{~V}$. The peak potential for the mixture (SP:TMAP:PPD is more positive than that required for the adsorption of an individual aryl diazonium cation: SP $(-0.47 \mathrm{~V})$, TMAP $(-0.26 \mathrm{~V})$ and PPD $(-0.36 \mathrm{~V})$. This shift in the peak potential suggests that, in mixed layers, the energy barriers for reducing diazonium cations may have decreased. A decrease in the energy barrier likely occurred between TMAP and PPD reductive peaks to yield a single peak at $-0.1 \mathrm{~V}$. The first peak at $-0.35 \mathrm{~V}$ may be attributed to SP diazonium with a small positive shift. Smaller reductive peak sizes with better passivation properties were observed for this mixture on the ITO surface compared with surfaces modified with either SP or TMAP alone. Similar results were also reported by authors of other studies of glassy carbon surfaces [26,27] (Fig. 1(1e)).

The reductive-adsorption cyclic voltammograms of the mixture of SP:TMAP:PABA showed a single reductive adsorption peak at approximately $-0.13 \mathrm{~V}$, which was likely associated with the reduction of PABA (Fig. 1(1f)). The dominance of PABA on the ITO surface is attributed to its lower negative reductive potential $(-0.1 \mathrm{~V})$ compared to those of SP $(-0.47 \mathrm{~V})$ and TMAP $(-0.26 \mathrm{~V})$. The passivation of the modified electrode surface (from the second cycle of modifications) may indicate that the rate of radical deposition on the ITO surface was much higher in this mixture compared with that on ITO surfaces modified with single species of radicals [27]. The reductive-adsorption voltammograms of the mixture of PABA and PPD showed single reductive adsorption peaks at approximately $-0.5 \mathrm{~V}$. The passivation of the ITO electrode surface was achieved after 5 cycles of surface modification. The mixed layer of the PABA- and PPD-modified ITO surface was further linked with the OEG aryl diazonium cation (Fig. 1(1g)). The PABA- and PPD/OEG-modified ITO surface was tested in both $\left[\mathrm{Fe}(\mathrm{CN})_{6}\right]^{4-/ 3-}$ and $\left[\mathrm{Ru}\left(\mathrm{NH}_{3}\right)_{6}\right]^{3+}$ redox-active solutions $(1 \mathrm{mM}, \mathrm{pH} 7)$. A moderate reduction in the cathodic and anodic peaks, respectively, was observed. This reduction may be due to the passivation effect of OEG, which is used in biomedical applications to provide an inert surface [32] (Fig. 1((2g) and (3g)). We expected that grafted ITO surfaces with SP:TMAP:PPD would repel both $\left[\mathrm{Fe}(\mathrm{CN})_{6}\right]^{4-/ 3-}$ and $\left[\mathrm{Ru}\left(\mathrm{NH}_{3}\right)_{6}\right]^{3+}$ because of the overall neutral charge of these mixtures on ITO surfaces. In the case of SP:TMAP:PABA mixtures, moderate passivation of the ITO electrode and reduced intensities of cathodic and anodic peaks were observed in the voltammograms of the $\left[\mathrm{Fe}(\mathrm{CN})_{6}\right]^{4-/ 3-}$ test solutions. However, no such effects were observed in the voltammograms of $\left[\mathrm{Ru}\left(\mathrm{NH}_{3}\right)_{6}\right]^{3+}$ test solutions. These results are attributable to the coating of ITO with a thin SP:TMAP:PABA layer, which has a moderate resistance to innersphere electron-transferring redox probes (e.g., resistance to the transfer of an electron through the formation of significant covalent bonding between the two species in a redox chemical reaction), such as $\left[\mathrm{Fe}(\mathrm{CN})_{6}\right]^{4-/ 3-}$ but a minimal barrier effect to redox probes that perform outer-sphere electron transfers (e.g., without covalent bonding), such as $\left[\mathrm{Ru}\left(\mathrm{NH}_{3}\right)_{6}\right]^{3+}$. Therefore, outer-sphere electron transfers are not affected.

\subsection{Antifouling study by CLSM}

\subsubsection{ITO surface modified with a mixture of SP:TMAP:PPD}

Comparisons between ITO surfaces functionalized with widely used antifouling molecules, OEG (ITO modified with PPD:PABA/OEG), and ITO surfaces functionalized with SP:TMAP:PPD at different molarity ratios of $1: 1: 0.37$ (mix 1), 1.5:0.5:0.37 $(\operatorname{mix} 2)$ and 0.5:1.5:0.37 ( $\operatorname{mix} 3$ ) were conducted in this study. An obviously lower fluorescence intensity was observed for SP:TMAP:PPD mixtures; the lowest fluorescence intensity was observed for a layer of mix 3 (Fig. 2). The important finding in this 

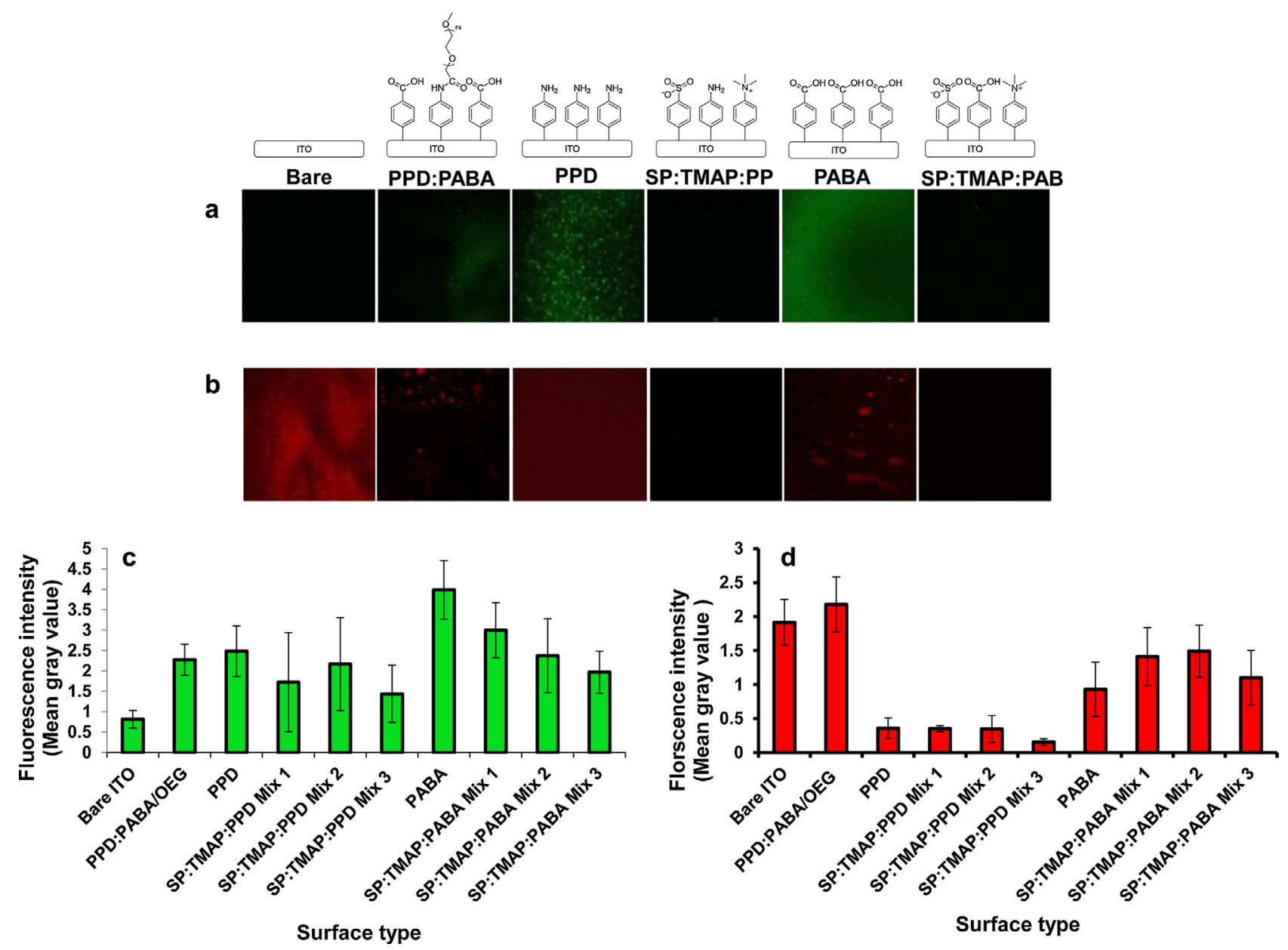

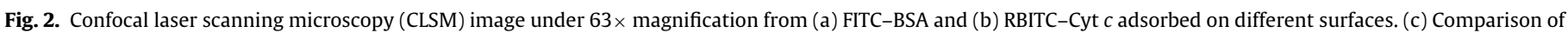
intensity of FITC-BSA adsorbed on different surfaces. (d) Comparison of intensity of RBITC-Cyt $c$ adsorbed on different surfaces.

study was that the ITO surface modified with SP:TMAP:PPD was capable of repelling both BSA-FITC and RBITC-Cyt $c$, as indicated by the low fluorescence intensities. (Note that percentages of fluorescent adsorptions for different ITO surfaces were calculated on the basis of the mean gray value, where all values were normalized to the ITO electrode modified with PPD:PABA/OEG.)

The fluorescence intensity percentage for adsorbed BSA-FITC on SP:TMAP:PPD-modified ITO surfaces for mixtures 1, 2 and 3 were lower than that for ITO functionalized with PPD:PABA/OEG. However, mix 3 exhibited the greatest resistance to BSA-FITC adsorption at $63 \%$ (a mean gray value of $1.4, s=0.7$ and $n=3$ ) compared with the $100 \%$ absorption of BSA-FITC in the control experiment (a mean gray value of $2.2, s=0.3$ and $n=3$ ), which involved an ITO surface functionalized with PPD:PABA/OEG (Table 1). The ITO modified with SP:TMAP:PPD mixtures 1,2 or 3 also exhibited excellent resistance to nonspecific protein adsorption. In this study, mixture 3 exhibited the best resistance because the fluorescence intensity percentage for the amount of adsorbed RBITC-Cyt $c$ on mix 3 was only $6.9 \%$ (a mean gray value of 0.15 , $s=0.05$ and $n=3$ ) compared with the $100 \%$ absorption of RBITC-Cyt $c$ of the control experiment (a mean gray value 2.1, $s=0.4$ and $n=3$ ), which involved functionalized ITO modified with PPD:PABA/OEG (Fig. 2).

Interestingly, the bare ITO exhibited the best capability to repel BSA-FITC, with a fluorescence intensity percentage of $35.6 \%$ and a mean gray value of only $0.81(s=0.2, n=3)$. In contrast, the bare ITO exhibited a high fluorescence intensity of $1.9(s=0.3, n=3)$
Table 1

The percentage of BSA-FITC and RBITC-Cyt $c$ adsorption at different surfaces represented by the normalized fluorescence intensities relative to ITO electrode modified with PPD:PABA/OEG.

\begin{tabular}{lcl}
\hline Surface type & $\begin{array}{c}\text { The percentage of the } \\
\text { fluorescent intensity } \\
\text { for adsorbed BSA-FITC }\end{array}$ & $\begin{array}{l}\text { The percentage of the } \\
\text { fluorescent intensity for } \\
\text { adsorbed RBITC-Cyt } c\end{array}$ \\
\hline Bare ITO & 35.68 & 87.89 \\
ITO/PPD & 109.54 & 16.45 \\
ITO/SP:TMAP:PPD with & 75.94 & 16.10 \\
molarity ratio of & & \\
1:1:0.37 (mix 1) & & \\
ITO/SP:TMAP:PPD with & 95.62 & \\
molarity ratio of & & \\
1.5:0.5:0.37 (mix 2) & & \\
ITO/SP:TMAP:PPD with & 63.33 & \\
molarity ratio of & & 42.86 \\
0.5:1.5:0.37 (mix 3) & & 64.68 \\
ITO/PABA & 175.64 & \\
ITO/SP:TMAP:PABA & 132.14 & 68.45 \\
with molarity ratio of & & \\
0.8:1:0.2 (mix 1) & & \\
ITO/SP:TMAP:PABA & 104.58 & \\
with molarity ratio of & & \\
1:1:0.1 (mix 2) & & \\
ITO/SP:TMAP:PABA & 86.72 & \\
with molarity ratio of & & \\
0.5:1:0.2 (mix 3) & & \\
ITO/PPD:PABA/OEG & 100.0 & \\
\hline
\end{tabular}


Table 2

XPS atomic ratio comparison of S, C, N between SP:TMAP:PPD-ITO and SP:TMAP:PABA-ITO.

\begin{tabular}{|c|c|c|c|c|c|c|}
\hline \multirow[t]{2}{*}{ Core level peaks } & \multirow{2}{*}{$\begin{array}{l}\text { S2p } \\
\text { S1 }\end{array}$} & \multicolumn{3}{|l|}{$\mathrm{C} 1 \mathrm{~s}$} & \multicolumn{2}{|l|}{ N1s } \\
\hline & & $\mathrm{C} 1$ & $\mathrm{C} 2$ & $\mathrm{C} 3$ & N1 & $\mathrm{N} 2$ \\
\hline SP: TMAP:PPD & 3.43 & 12.1 & 25.7 & - & 0.64 & 2.75 \\
\hline SP: MAP:PABA & 0.41 & 18.9 & 8.5 & 2.9 & 1.0 & 0.6 \\
\hline
\end{tabular}
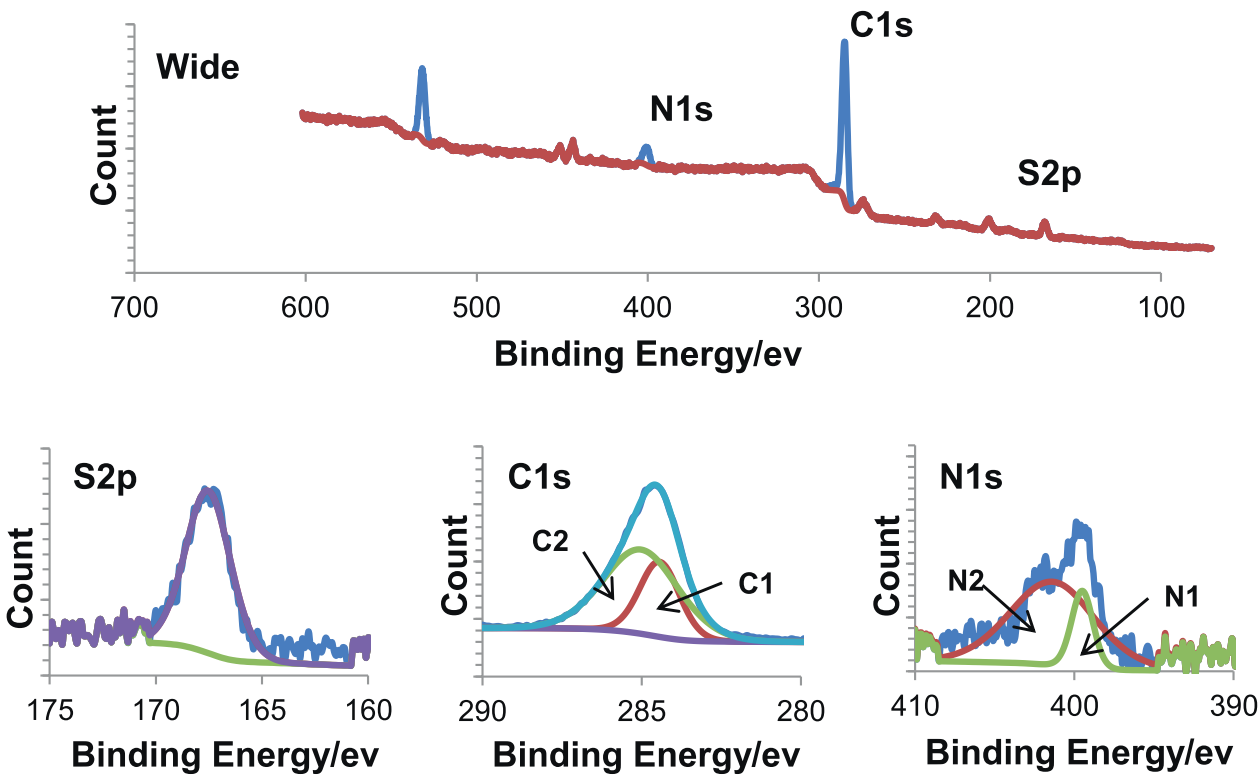

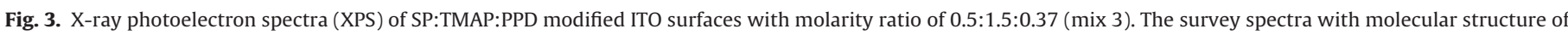
S2p, C1s and N1s core-level spectra.

after adsorbing RBITC-Cyt $c$. This observation is explained by the chemical composition of the ITO surfaces, which consist of negatively charged complexes of stoichiometric oxide, hydroxides, oxyhydroxides, and physisorbed indium hydroxides. Hence, these negative charges may repel BSA-FITC but increase the adsorption of positively charge proteins such as RBITC-Cyt $c$ [22].

In conclusion, a surface modified with zwitterionic or similar molecules exhibited better antifouling properties in repelling BSA-FITC and RBITC-Cyt $c$ compared with ITO/PPD:PABA/OEG. This better antifouling behavior may be due to good packing and charge balancing of zwitterionic groups on the ITO modified with SP:TMAP:PPD mixtures. The ITO surface modified with SP:TMAP:PPD (mix 3 ) showed the best resistance to nonspecific adsorptions of BSA-FITC and RBITC-Cyt $c$ compared with mix 1 and mix 2. We attribute the differences in the antifouling capability of SP:TMAP:PPD mixtures to the electrostatic interaction between the three molecules used to fabricate the inert surfaces. At this ratio, the electrostatic interaction between the positively charged tail group (TMAP:PPD) and the negatively charged tail group (SP) appear to provide the best electrically neutral electronic structure.
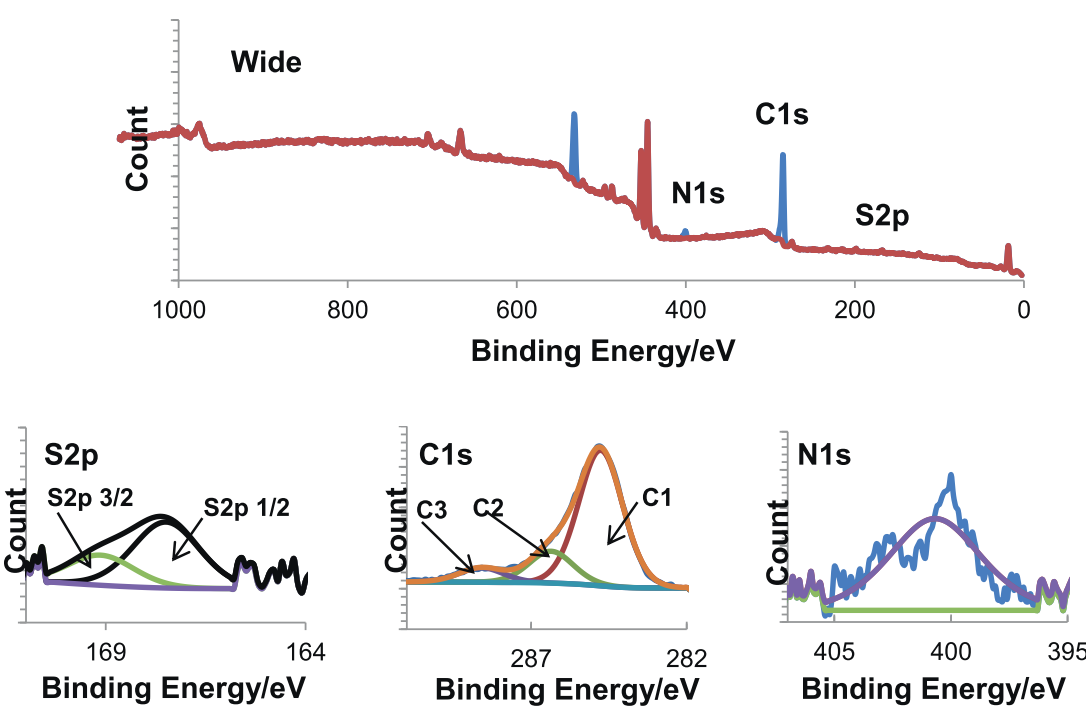

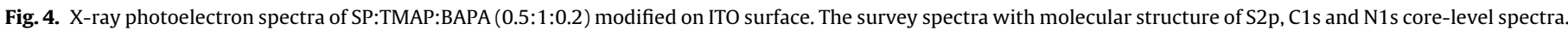




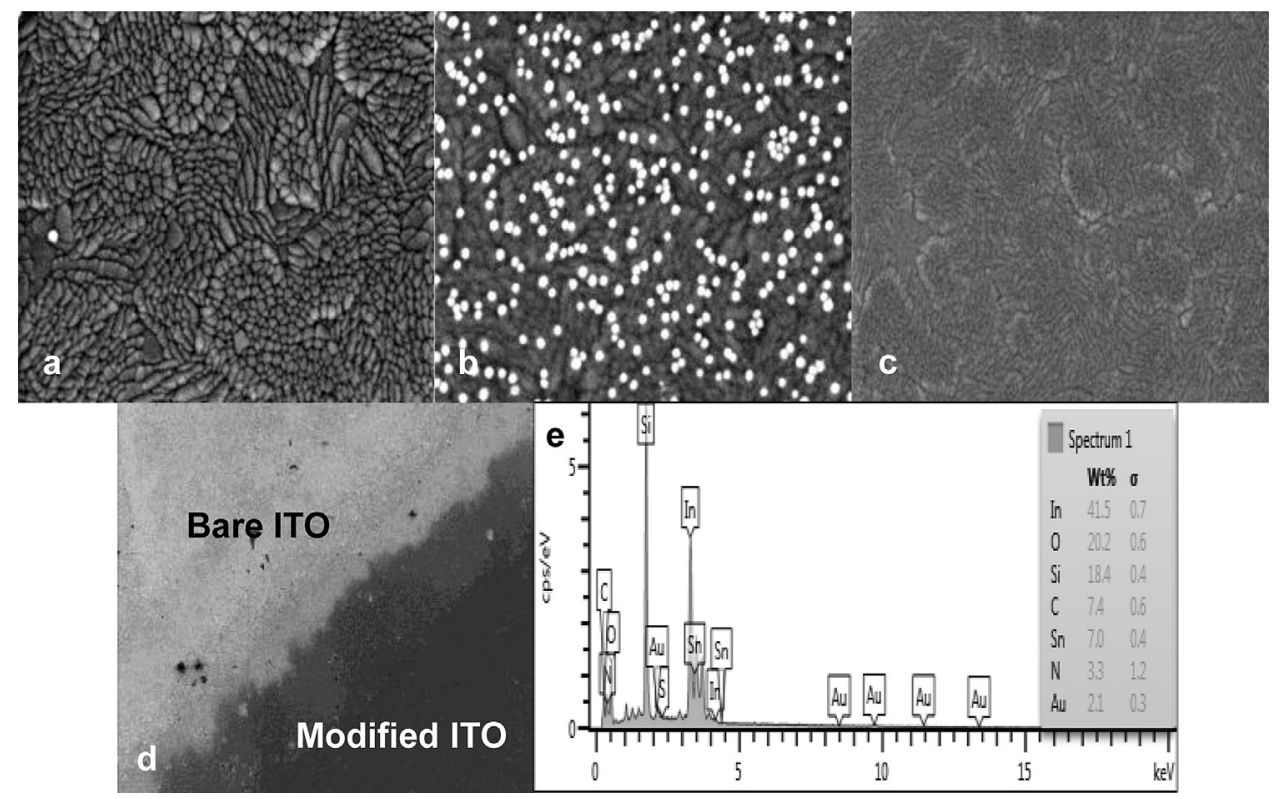

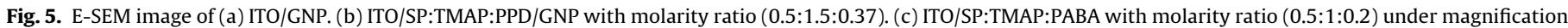
50 K. (d) ITO/SP:TMAP:PABA and bare ITO surface under magnification $1.5 \mathrm{~K}$. (e) EDX spectra ITO/SP:TMAP:PPD/GNP.

\subsubsection{ITO surface modified with a mixture of SP:TMAP:PABA}

ITO surfaces were modified with SP:TMAP:PABA at different molarity ratios of $0.8: 1: 0.2(\operatorname{mix} 1), 1: 1: 0.1(\operatorname{mix} 2)$ and $0.5: 1: 0.2$ (mix 3). Mix 3 exhibited the best antifouling capabilities among the three combinations against both BSA-FITC (86.7\% fluorescent intensity, a mean gray value of $1.96 ; s=0.5, n=3$ ) and RBITC-Cyt $c(50.5 \%$ fluorescent intensity, a mean gray value of $1.1 ; s=0.4$, $n=3$ ) and compared with the ITO surface functionalized with PPD:PABA/OEG (100\% BSA-FITC fluorescent intensity, a mean gray value of $2.2 ; s=0.3, n=3 ; 100 \%$ RBITC-Cyt $c$ fluorescent intensity, a mean gray value of $2.1 ; s=0.4, n=3$; Fig. 2 ; Table 1 ).

The ITO electrode modified with PPD:PABA/OEG accumulated a significant amount of RBITC-Cyt $c$. It exhibited the highest fluorescence intensity among the investigated surface types (Fig. 2(d)). RBITC-Cyt $c$ has the ability to change its conformation upon adsorption onto a wide range of interfaces $[33,34]$. Furthermore, the failure of OEG moieties to resist the nonspecific adsorption of undesired proteins may be due to the auto-oxidation of OEG moieties [15,35], which makes the PPD:PABA/OEG layer unable to repel BSA-FITC and RBITC-Cyt $c$. Antifouling coating alternatives that are more effective than OEG should therefore be identified.

ITO modified with SP:TMAP:PPD mixtures exhibited better resistance to the nonspecific adsorption of BSA-FITC and RBITC-Cyt $c$ compared with SP:TMAP:PABA. This better resistance may be due to the domination of PABA molecules in SP:TMAP:PABA mixtures (Fig. 1(1f)). The adsorption of aryl diazonium cation mixtures was dominated by the aryl diazonium cation, which was easier to reduce (e.g., having a less negative potential) [36]; such behavior subsequently reduced adsorptions of the other two molecules (e.g., SP and TMAP, which were necessary for a neutral charge interface. In the SP:TMAP:PPD mixture, no such domination was observed for the PPD molecule. XPS analysis showed that the atomic ratio for SP and TMAP was higher in SP:TMAP:PPD than in SP:TMAP:PABA mixtures (Table 2).

In contrast to PABA, PPD could be used as a linker molecule and be incorporated with zwitterionic molecules (e.g., SP and TMAP) to resist the nonspecific adsorption of BSA-FITC and RBITC-Cyt c. PPD was more readily deposited onto the ITO surface, and the complete passivation of the electrode surface required only two cycles (Fig. 1(1c)). With PABA, the passivation was significantly less efficient, requiring 10 cycles (Fig. 1(1d)). PPD alone can resist the nonspecific adsorption of BSA-FITC and RBITC-Cyt $c$ (Fig. 1, Table 1). Such behavior enhanced the antifouling efficiency of zwitterionic molecules (e.g., SP and TMAP). Moreover, the reductive adsorption potential for PPD was $-0.36 \mathrm{~V}$, which, in comparison to the potential for PABA $(-0.1 \mathrm{~V})$, was closer to reductive adsorptions of SP $(-0.473 \mathrm{~V})$ and TMAP $(-0.26 \mathrm{~V})$. Therefore, aryl diazonium cations with similar reductive adsorption potentials prevented the domination of one aryl diazonium cation over the other, which aided the fabrication of a neutrally charged antifouling coating.

\subsection{ITO surface characterization via XPS analysis}

For ITO surfaces modified with SP:TMAP:PPD (mix 3), the presence of SP was confirmed by a signal peak indicative of sulfur (S2p) at $167.9 \mathrm{eV}[27,28]$. This signal peak was absent in the narrow scan of the S2p region for bare ITO (Fig. 3). TMAP was detected by the presence of a C-N linkage peak, which represents the link between nitrogen and 4-methyl groups, at a binding energy of $285.0 \mathrm{eV}$ (C2). Another peak (C1) centered at $284.4 \mathrm{eV}$ in a narrow scan of the carbon region represented aromatic carbons in SP, PPD and TMAP moieties [27,30]. PPD was characterized by the $\mathrm{N} 1399.5 \mathrm{eV}$ peak observed in the narrow scan of the N1s region, which is attributed to $\mathrm{H}_{2} \mathrm{~N}-\mathrm{C}[23-25,37]$. In contrast, XPS survey spectra for the mixed SP:TMAP:PABA layer (Fig. 4) revealed a peak at $167.8 \mathrm{eV}$ corresponding to S2p, which indicated an SP graft $[23,27,28]$. A narrow scan of the carbon $\mathrm{C} 1 \mathrm{~s}$ region revealed three peaks centered at $284.7 \mathrm{eV}(\mathrm{C} 1), 286.1 \mathrm{eV}(\mathrm{C} 2)$, and $288.5 \mathrm{eV}(\mathrm{C} 3)$. The $\mathrm{C} 1$ and $\mathrm{C} 2$ peaks were attributed to aromatic carbon atoms and to $\mathrm{N}-\left(\mathrm{CH}_{3}\right)_{3}$ in the trimethylammonio group, respectively. The $\mathrm{C} 3$ peak corresponded to $\mathrm{COO}^{-}$; the presence of this peak indicates the successful grafting of PABA molecules onto the ITO substrate [23,38,39].

\subsubsection{ITO surface characterization via FE-SEM analysis}

An FE-SEM image of the bare ITO electrode showed the absence of GNP (Fig. 5(a)). However when the ITO was modified with SP:TMAP:PPD, a uniform and homogenous distribution of GNPS was observed (Fig. 5(b)). The bare ITO/GNP experiment as shown in Fig. 5(a) served as a control experiment. The objective was to demonstrate that the attachment of GNPs onto the ITO surface 
only formed with the present of PPD. The EDX results confirmed the deposition of GNPs onto modified ITO electrode surfaces at an atomic ratio of 2.11 (Fig. 5(e)). The linkage of amines in the mixture of SP:TMAP:PPD to GNPs was more likely to occur by dehydrogenation from NH-GNP because of its stronger covalent bond [24,40]. Such observations confirmed the homogenous grafting of the linker molecule (PPD) onto the ITO surface, unlike the SP:TMAP:PABA mixture, which was dominated with PABA molecule (XPS results are shown in Table 2). The image of an SP:TMAP:PABA-modified ITO electrode showed a thin, smooth and featureless morphology indicates that no GNPs could be deposited onto this type of surface (Fig. 5(c)). An obvious difference was noticed in images between the bare and SP:TMAP:PABA modified ITO surfaces which captured at $1500 \times$ magnification (Fig. 5(d)).

\section{Conclusions}

A biosensing interface consisting of antifouling and linker molecules based on in-situ-generated aryl diazonium cations was described. The differences in electrochemical behaviors among all types of modified surfaces (either with the individual compound or a mixed layer of aryl diazonium cations) were investigated and evaluated. An antifouling study using confocal laser scanning microscopy showed that ITO surfaces coated with mixed layers of SP:TMAP:PPD 0.5:1.5:0.37 ( $\operatorname{mix} 3$ ) or SP:TMAP:PABA 0.5:1:0.2 ( $\mathrm{mix} 3$ ) exhibited better antifouling capabilities against both BSA-FITC and RBITC-Cyt $c$ compared to ITO surfaces coated with PPD:PABA/OEG. However, PPD:PABA/OEG-coated ITO surfaces showed lower amounts of BSA-FITC adsorbed compared to SP:TMAP:PABA-coated ITO surfaces. The mixture of SP:TMAP:PPD showed better resistance to nonspecific adsorptions of BSA-FITC and RBITC-Cyt $c$ compared to SP:TMAP:PABA. Analyses of XPS survey spectra further confirmed the deposition of SP:TMAP:PPD and SP:TMAP:PABA onto ITO surfaces. FE-SEM and EDX analyses showed dense and homogenous distributions of GNPs, which indicated a good distribution of linker and antifouling molecules on the ITO sensing interfaces. This study highlights the prospective use of aryl-diazonium-cation-derived zwitterionic compounds and linker molecules for the easy fabrication of ITO biosensing interfaces. These interfaces can repel undesirable anionic and cationic proteins and detect target analytes in highly complicated biological matrices, such as blood, serum, and urine.

\section{Acknowledgments}

This work was financially supported by the University of Malaya Research Grant (UMRG) (RG159-12SUS, RP012C-14SUS), the Fundamental Research Grant Scheme (FRGS) from the Ministry of Higher Education of Malaysia (MOHE) (FP014-2013A), a High Impact Research Grant from the Ministry of Higher Education of Malaysia (HIR-MoHE F000004-21001), and University of Malaya Postgraduate Research Grant (PG120-2012B).

\section{References}

[1] D. Atias, Y. Liebes, V. Chalifa-Caspi, L. Bremand, L. Lobel, R.S. Marks, P. Dussart, Chemiluminescent optical fiber immunosensor for the detection of IgM antibody to dengue virus in humans, Sens. Actuators, B: Chem. 140 (2009) 206-215.

[2] I.T. Cavalcanti, B.V.M. Silva, N.G. Peres, P. Moura, M.D.P.T. Sotomayor, M.I.F. Guedes, R.F. Dutra, A disposable chitosan-modified carbon fiber electrode for dengue virus envelope protein detection, Talanta 91 (2012) 41-46.

[3] W.H. Chen, I.H. Hsu, Y.C. Sun, Y.K. Wang, T.K. Wu, Immunocapture couples with matrix-assisted laser desorption/ionization time-of-flight mass spectrometry for rapid detection of Type 1 dengue virus, J. Chromatogr. A 1288 (2013) 21-27.

[4] A.C.M.S. Dias, S.L.R. Gomes-Filho, M.M.S. Silva, R.F. Dutra, A sensor tip based on carbon nanotube-ink printed electrode for the dengue virus NS1 protein, Biosens. Bioelectron. 44 (2013) 216-221.
[5] M.J.A. Shiddiky, P.H. Kithva, D. Kozak, M. Trau, An electrochemical immunosensor to minimize the nonspecific adsorption and to improve sensitivity of protein assays in human serum, Biosens. Bioelectron. 38 (2012) 132-137.

[6] P. Akkahat, S. Kiatkamjornwong, i.S. Yusa, V.P. Hoven, Y. Iwasaki, Development of a novel antifouling platform for biosensing probe immobilization from methacryloyloxyethyl phosphorylcholine-containing copolymer brushes, Langmuir 28 (2012) 5872-5881.

[7] S. Salomon, T. Leichle, D. Dezest, F. Seichepine, S. Guillon, C. Thibault, C. Vieu, L. Nicu, Arrays of nanoelectromechanical biosensors functionalized by microcontact printing, Nanotechnology 23 (2012) 495501.

[8] M. Gabriel, D. Strand, C.-F. Vahl, Cell adhesive and antifouling polyvinyl chloride surfaces via wet chemical modification, Artif. Organs 36 (2012) 839-844.

[9] N. Wang, K. Burugapalli, W. Song, J. Halls, F. Moussy, A. Ray, Y. Zheng, Electrospun fibro-porous polyurethane coatings for implantable glucose biosensors, Biomaterials 34 (2013) 888-901.

[10] K.H. Chae, Y.M. Jang, Y.H. Kim, O.-J. Sohn, J.I. Rhee, Anti-fouling epoxy coatings for optical biosensor application based on phosphorylcholine, Sens. Actuators, B: Chem. 124 (2007) 153-160.

[11] S. Brahim, D. Narinesingh, A. Guiseppi-Elie, Bio-smart hydrogels: co-joined molecular recognition and signal transduction in biosensor fabrication and drug delivery, Biosens. Bioelectron. 17 (2002) 973-981.

[12] S. Zhang, G. Wright, Y. Yang, Materials and techniques for electrochemical biosensor design and construction, Biosens. Bioelectron. 15 (2000) 273-282.

[13] Q. Yu, Y. Zhang, H. Wang, J. Brash, H. Chen, Anti-fouling bioactive surfaces, Acta Biomater. 7 (2011) 1550-1557.

[14] P.-Y.J. Yeh, J.N. Kizhakkedathu, J.D. Madden, M. Chiao, Electric field and vibration-assisted nanomolecule desorption and anti-biofouling for biosensor applications, Colloids Surf., B: Biointerfaces 59 (2007) 67-73.

[15] A.L. Gui, E. Luais, J.R. Peterson, J.J. Gooding, Zwitterionic phenyl layers: finally, stable, anti-biofouling coatings that do not passivate electrodes, ACS Appl. Mater. Interfaces 5 (2013) 4827-4835.

[16] J.E. Gittens, T.J. Smith, R. Suleiman, R. Akid, Current and emerging environmentfriendly systems for fouling control in the marine environment, Biotechnol. Adv. 31 (2013) 1738-1753

[17] Q. Shi, Y. Su, W. Chen, J. Peng, L. Nie, L. Zhang, Z. Jiang, Grafting short-chain amino acids onto membrane surfaces to resist protein fouling, J. Membr. Sci. 366 (2011) 398-404.

[18] S.S. Shah, M.C. Howland, L.-J. Chen, J. Silangcruz, S.V. Verkhoturov, E.A. Schweikert, A.N. Parikh, A. Revzin, Micropatterning of proteins and mammalian cells on indium tin oxide, ACS Appl. Mater. Interfaces 1 (2009) 2592-2601.

[19] M.J.A. Shiddiky, P.H. Kithva, R. Sakandar, M. Trau, Femtomolar detection of a cancer biomarker protein in serum with ultralow background current by anodic stripping voltammetry, Chem. Commun. 48 (2012) 6411-6413.

[20] Y. Li, M. Giesbers, M. Gerth, H. Zuilhof, Generic top-functionalization of patterned antifouling zwitterionic polymers on indium tin oxide, Langmuir 28 (2012) 12509-12517.

[21] P. Liu, Z. Su, Surface-initiated atom transfer radical polymerization (SI-ATRP) of styrene from chitosan particles, Mater. Lett. 60 (2006) 1137-1139.

[22] M. Chockalingam, N. Darwish, G. Le Saux, J.J. Gooding, Importance of the indium tin oxide substrate on the quality of self-assembled monolayers formed from organophosphonic acids, Langmuir 27 (2011) 2545-2552.

[23] G. Liu, M. Chockalingham, S.M. Khor, A.L. Gui, J.J. Gooding, A comparative study of the modification of gold and glassy carbon surfaces with mixed layers of in situ generated aryl diazonium compounds, Electroanalysis 22 (2009) 918-926.

[24] G. Liu, E. Luais, J.J. Gooding, The fabrication of stable gold nanoparticle-modified interfaces for electrochemistry, Langmuir 27 (2011) 4176-4183.

[25] S. Eissa, C. Tlili, L. L'Hocine, M. Zourob, Electrochemical immunosensor for the milk allergen $\beta$-lactoglobulin based on electrografting of organic film on graphene modified screen-printed carbon electrodes, Biosens. Bioelectron. 38 (2012) 308-313.

[26] A.L. Gui, H.M. Yau, D.S. Thomas, M. Chockalingam, J.B. Harper, J.J. Gooding, Using supramolecular binding motifs to provide precise control over the ratio and distribution of species in multiple component films grafted on surfaces: demonstration using electrochemical assembly from aryl diazonium salts, Langmuir 29 (2013) 4772-4781.

[27] N. Vila, D. Belanger, Mixtures of functionalized aromatic groups generated from diazonium chemistry as templates towards bimetallic species supported on carbon electrode surfaces, Electrochim. Acta 85 (2012) 538-547.

[28] N. Vila, M.V. Brussel, M. DAmours, J. Marwan, C. Buess-Herman, D. Belanger Metallic and bimetallic $\mathrm{Cu} / \mathrm{Pt}$ species supported on carbon surfaces by means of substituted phenyl groups, J. Electroanal. Chem. 609 (2007) 85-93.

[29] C. Combellas, F. Kanoufi, J. Pinson, F.I. Podvorica, Sterically hindered diazonium salts for the grafting of a monolayer on metals, J. Am. Chem. Soc. 130 (2008) 8576-8577.

[30] J. Lyskawa, D. Belanger, Direct modification of a gold electrode with aminophenyl groups by electrochemical reduction of in situ generated aminophenyl monodiazonium cations, Chem. Mater. 18 (2006) 4755-4763.

[31] R. Polsky, J.C. Harper, D.R. Wheeler, S.M. Dirk, D.C. Arango, S.M. Brozik Electrically addressable diazonium-functionalized antibodies for multianalyte electrochemical sensor applications, Biosens. Bioelectron. 23 (2008) 757-764.

[32] W.-P. Hu, L.-Y. Huang, T.-C. Kuo, W.-W. Hu, Y. Chang, C.-S. Chen, H.-C Chen, W.-Y. Chen, Optimization of DNA-directed immobilization on mixed oligo(ethylene glycol) monolayers for immunodetection, Anal. Biochem. 423 (2012) 26-35 
[33] X. Chen, R. Ferrigno, J. Yang, G.M. Whitesides, Redox Properties of cytochrome $c$ adsorbed on self-assembled monolayers: a probe for protein conformation and orientation, Langmuir 18 (2002) 7009-7015.

[34] D. Hobara, S.-i. Imabayashi, T. Kakiuchi, Preferential adsorption of horse heart cytochrome $c$ on nanometer-scale domains of a phase-separated binary selfassembled monolayer of 3-mercaptopropionic acid and 1-hexadecanethiol on $\mathrm{Au}(111)$, Nano Lett. 2 (2002) 1021-1025.

[35] R.E. Holmlin, X. Chen, R.G. Chapman, S. Takayama, G.M. Whitesides, Zwitterionic SAMs that resist nonspecific adsorption of protein from aqueous buffer, Langmuir 17 (2001) 2841-2850.

[36] C. Louault, M. D’Amours, D. Bélanger, The electrochemical grafting of a mixture of substituted phenyl groups at a glassy carbon electrode surface, ChemPhysChem 9 (2008) 1164-1170.
[37] I. Losito, C. Malitesta, I. De Bari, C.-D. Calvano, X-ray photoelectron spectroscopy characterization of poly(2, 3-diaminophenazine) films electrosynthesised on platinum, Thin Solid Films 473 (2005) 104-113.

[38] D.-J. Chung, S.-H. Oh, S. Komathi, A.I. Gopalan, K.-P. Lee, S.-H. Choi, One-step modification of various electrode surfaces using diazonium salt compounds and the application of this technology to electrochemical DNA (E-DNA) sensors, Electrochim. Acta 76 (2012) 394-403.

[39] M. Yin, Y. Yuan, C. Liu, J. Wang, Development of mussel adhesive polypeptide mimics coating for in-situ inducing re-endothelialization of intravascular stent devices, Biomaterials 30 (2009) 2764-2773.

[40] G. Liu, J. Liu, T.P. Davis, J.J. Gooding, Electrochemical impedance immunosenso based on gold nanoparticles and aryl diazonium salt functionalized gold electrodes for the detection of antibody, Biosens. Bioelectron. 26 (2011)3660-3665 